Open Access

Natalia Morollón Martí*

\title{
El potencial pedagógico de la pragmática sociocultural como herramienta de mediación en la interpretación de experiencias interculturales
}

\section{The pedagogical potential of sociocultural pragmatics as a mediational tool for interpreting intercultural experiences}

DOI 10.1515/soprag-2017-0009

Resumen: El propósito de este trabajo es investigar de qué forma una aproximación sociocultural al estudio de la cortesía puede contribuir a promover la conciencia metapragmática e introducir una dimensión intercultural en el aula universitaria de español como lengua extranjera (ELE). Para ello, esta investigación muestra el análisis de la actividad reflexiva en grupos de estudiantes universitarios. Durante el desarrollo de estas reflexiones, el conocimiento teórico y conceptual relativo al área de investigación de la pragmática sociocultural, presentado en forma de enseñanza explícita, es empleado como herramienta de mediación durante el desarrollo del diálogo colaborativo (Swain, 2006. Languaging, agency and collaboration in advanced second language profiency. En H. Byrnes (Ed.), Advanced Language Learning: The Contribution of Halliday and Vygotsky (pp. 95-108). London: Continuum) en la interpretación de sus experiencias interculturales, obtenidas en sesiones de telecolaboración con hablantes nativos de español. El análisis cualitativo de los datos muestra cómo la presentación de conceptos teóricos, en lugar de mostrar normas generalizadas relativas al uso de la lengua, permite a los estudiantes participar en una actividad en la que construyen el significado de lo sucedido durante la interacción verbal a través del significado conceptual. Asimismo, esta aproximación sociocultural aplicada estudio de la cortesía ofrece oportunidades

*Corresponding author: Natalia Morollón Martí, Syddansk Universitet, Odense 5230, Denmark, E-mail: nmorollon@hotmail.com 
de interpretación de experiencias interculturales desde una perspectiva relativista en la que se consideran los factores sociales y culturales específicos de cada comunidad de habla estudiada y permite realizar comparaciones sobre el uso de la lengua en la interacción intercultural. Estas reflexiones y comparaciones son aspectos fundamentales en el desarrollo de la comprensión intercultural.

Palabras clave: enseñanza y aprendizaje de pragmática de L2, conciencia metapragmática, diálogo colaborativo, pragmática sociocultural

Abstract: The purpose of this study is to investigate in which way a sociocultural approach to the study of politeness can contribute to promote metapragmatic awareness and introduce an intercultural dimension in the Spanish as Foreign Language university classroom. Therefore, this investigation shows the analysis of the reflexive activity among groups of university students. During these reflections, both the theoretical and conceptual knowledge related to the field of sociocultural pragmatics, presented to the students as explicit instruction, is used as mediational tool during collaborative dialogue (Swain, 2006. Languaging, agency and collaboration in advanced second language profiency. En H. Byrnes (Ed.), Advanced Language Learning: The Contribution of Halliday and Vygotsky (pp. 95-108). London: Continuum) in the interpretation of their intercultural experiences throughout telecollaborative sessions with native speakers of Spanish. The qualitative analysis of the data shows how the presentation of theoretical concepts, instead of showing generalized norms related to language use, allows students to take part in an activity where they construct the meaning of what happened during verbal interaction through conceptual meaning. This sociocultural approach to the study of politeness offers opportunities for understanding intercultural experiences from a relativist perspective, in which social and cultural specific factors from the speech communities under study are considered, and enables students to make comparisons about the language use in intercultural communication. These reflections and comparisons are essential in the development of intercultural understanding.

Keywords: L2 pragmatics teaching and learning, metapragmatic awareness, collaborative dialogue, sociocultural pragmatics

\section{Introducción}

El proceso de globalización de los últimos años junto con la digitalización de las prácticas comunicativas ha traído consigo una considerable ampliación del 
contacto entre personas de diferentes culturas (Thorne, 2003). Ante esta realidad, y en respuesta a las demandas de una sociedad multicultural, en el área de enseñanza y aprendizaje de segundas lenguas (L2) se ha destacado la necesidad de acentuar la confluencia existente entre el uso de la lengua y la cultura (Kramsch, 1993; Byram, 2008). Así, se ha llevado a cabo una reconceptualización en cuanto a lo que se considera debe ser el objeto principal de enseñanza, conocida como "the intercultural turn" (Thorne, 2010), donde el foco de atención ha pasado de estar en los aspectos meramente lingüísticos y comunicativos de la lengua a centrarse en una dimensión intercultural de la misma. Esta nueva aproximación a la enseñanza de L2 ha sido considerada beneficiosa al prevenir la formación de estereotipos y evitar malentendidos en las relaciones interculturales (Thorne, 2010; Cohen y Sykes, 2013). En este contexto, la pragmática, definida por Ishihara y Cohen (2010) como el área de intersección entre lengua y cultura, adquiere una posición central en ámbitos de enseñanza de L2. Esto se debe a que permite introducir en el aula aspectos culturales sin perder de vista la dimensión lingüística (Dervin y Liddicoat, 2013).

Estudios centrados en la enseñanza y aprendizaje de pragmática de L2 han subrayado la enseñabilidad de esta disciplina lingüística y la importancia de integrarla en el aula de L2 (Kasper y Rose, 2002; Alcón Soler y Martínez-Flor, 2008). Además, se han mostrado los beneficios de la instrucción frente a la no instrucción o la simple exposición a la L2 y la utilidad de aplicar métodos explícitos de enseñanza acompañada de información metapragmática (Taguchi, 2015). Sin embargo, sin negar el aporte de estas investigaciones, estos estudios han centrado la enseñanza y evaluación de pragmática en L2 en la producción de determinados actos de habla en base a un modelo de hablante nativo a partir del cual determinar el grado de adecuación en el uso de las normas pragmáticas. Considerando la problemática que presenta el empleo de estos criterios al no poder estimar a los hablantes nativos como un grupo homogéneo (Hymes, 1972; Kramsch, 1993; van Compernolle, 2014), se propone en este trabajo dirigir el foco de atención de la enseñanza y aprendizaje de pragmática de L2 hacia el desarrollo de la conciencia metapragmática desde una dimensión intercultural del uso de la lengua.

Con la intención de promover la conciencia intercultural en contextos de aprendizaje de L2, diferentes autores (Kramsch, 1993; Liddicoat y Scarino, 2013) indican la importancia de reconocer como uno de los objetivos principales el desarrollo de hábitos de observación, comparación, reflexión e interpretación sobre la propia cultura (C1), la cultura meta (C2) y el significado que el uso del lenguaje adquiere en ambas. Además, y debido a que estas reflexiones y comparaciones no siempre se producen de forma espontánea por los aprendices, se subraya la necesidad de desarrollar prácticas pedagógicas guiadas por el 
profesor que puedan llegar a automatizarse y emplearse en diferentes contextos comunicativos. Lo fundamental, consideran estos autores, no es evaluar estas interpretaciones en términos de adecuación o inadecuación, sino crear espacios de reflexión donde el objetivo principal del aprendizaje sea la actividad de interpretación intercultural en sí. Así, ya que el lenguaje es una parte constituyente de la cultura, reflexionar sobre su uso puede ser el punto de partida en la realización de reflexiones en las que se identifican las similitudes y diferencias de su uso en diferentes prácticas comunicativas (Dervin y Liddicoat, 2013). Por su parte, McConachy indica cómo el uso del habla metapragmática constituye la base a partir de la que establecer relaciones entre lengua y cultura, y señala: "meta-pragmatic awareness for intercultural communication can be understood on a fundamental level, as a view of language as a contextually contingent social tool in which individuals orient to pragmatic phenomena based on culturally situated frames of reference” (Mc Conachy, 2013, p. 102).

Esta concepción intercultural de la enseñanza de pragmática de L2 se aleja de la visión estática del uso de la lengua evaluada en base a un modelo ideal de habla nativo. A este respecto, van Compernolle (2014) problematiza sobre la ambigüedad que supone utilizar el concepto de adecuación por no poder determinar hasta qué punto un enunciado es adecuado o inadecuado en situaciones en las que podrían encontrarse argumentos válidos para el uso de otras formas lingüísticas. Aun reconociendo la importancia de que los aprendices de L2 conozcan la existencia de las convenciones sociales y culturales compartidas que se manifiestan mediante el uso del habla, este autor considera necesario reconocer la agentividad del aprendiz de L2 como hablante. Así, se hace fundamental respetar el significado específico que se desea transmitir en una determinada situación comunicativa, que puede coincidir, o no, con las normas sociales consideradas representativas. Además, desde esta perspectiva intercultural, se admite que los pensamientos, sentimientos y valores de los aprendices no pueden ser dejados de lado para adaptarse a los de la lengua meta (Liddicoat, 2008). Esto se debe a que, en algunas ocasiones, su uso puede contradecir aspectos relacionados con su identidad, principios o personalidad (Kinginger y Farrell, 2004; Kinginger, 2008).

Considerando estas premisas, y como forma de desarrollar prácticas reflexivas en el aula de L2, se propone en este trabajo introducir a los estudiantes de ELE a una perspectiva teórica pragmática de relatividad cultural del uso de la lengua, que les permita realizar interpretaciones contextualizadas del intercambio verbal intercultural en el que participan. De forma específica, esta propuesta consiste en: (a) introducir a los estudiantes, en forma de enseñanza explícita, al estudio de la cortesía como fenómeno social y comunicativo desde una aproximación sociocultural, área de estudio conocida como pragmática 
sociocultural (Bravo, 2002; Bravo, 2004; Bravo y Briz, 2004); y (b) fomentar el desarrollo de hábitos de reflexión, donde el conocimiento teórico y conceptual sea empleado en la interpretación de diferentes situaciones comunicativas, tanto en la lengua nativa como en la L2 estudiada. A partir de esta propuesta, en este estudio se muestra el análisis de los datos recogidos durante las reflexiones realizadas en grupos en una intervención didáctica orientada al desarrollo de la concienciación sociopragmática entre estudiantes daneses universitarios de ELE (Morollón Martí y Fernández, 2016). La contribución de esta investigación es doble. Por un lado, contribuye a mostrar de qué forma la enseñanza de pragmática de L2 puede integrarse en un plan curricular universitario, necesidad planteada por Gironzetti y Koike (2016). Por otro lado, muestra cómo la enseñanza de la pragmática sociocultural en el aula de ELE permite desarrollar la conciencia metapragmática de los estudiantes a la vez que realizar interpretaciones interculturales desde una perspectiva de relatividad cultural.

La estructura seguida en este trabajo es la siguiente: en el apartado 2 se delimitará la conciencia metapragmática desde la aproximación teórica adoptada en este estudio, la teoría sociocultural ${ }^{1}$ (TSC), y se introducirán los principios y conceptos más relevantes relativos al estudio de la cortesía desde una perspectiva sociocultural. En el apartado 3 se describirá el marco metodológico, el contexto en el que se recogieron los datos y el procedimiento de análisis empleado. Finalmente, en el apartado 4 se mostrará el análisis y discusión de los datos, y en el apartado 5 se expondrán las conclusiones de este estudio.

\section{Concienciación metapragmática en L2: uso del lenguaje y de los conceptos como herramientas de mediación cognitiva}

En un intento de alejarse de una concepción estática de la enseñanza y el aprendizaje de pragmática de L2 centrada en el uso de las normas pragmáticas por parte de

1 Debe diferenciarse en este estudio entre el uso del término sociocultural en relación con la pragmática sociocultural, relativo a los aspectos sociales y culturales de un contexto determinado, y la teoría sociocultural (TSC) propuesta por Vygotsky (1978). En esta teoría, también conocida con el nombre de psicología histórico-cultural, el empleo del término sociocultural está relacionado con la concepción que en ella se presenta del desarrollo humano, el cual se entiende como una actividad mediada por herramientas psicológicas construidas culturalmente como el lenguaje o los conceptos y que, además, tiene lugar durante la interacción social con otras personas. 
los estudiantes de L2, se defiende en este trabajo centrar esta enseñanza en el desarrollo de la conciencia metapragmática. De esta forma, el objetivo principal es fomentar la comprensión de las normas pragmáticas estudiadas por medio de prácticas reflexivas y alcanzar una mayor comprensión de las normas sociales y culturales que se manifiestan en la interacción verbal. Siguiendo la definición propuesta por Kinginger y Farrell (2004), la concienciación metapragmática es "knowledge of the social meaning of variable second language (L2) forms and awareness of the ways in which these forms mark different aspects of social contexts" (p. 20). El desarrollo de esta habilidad entre los estudiantes de L2, como señala Mc Conachy (2013), supone promover la comprensión e interpretación del propósito comunicativo en la realización de diferentes formas lingüísticas y establecer relaciones entre la realización de estas formas lingüísticas y su contexto de uso.

\subsection{Aproximación teórica al aprendizaje}

En este trabajo se parte de una aproximación teórica sociocultural (TSC) (Vygotsky, 1978, 1986) aplicada al área de aprendizaje de L2 (Lantolf y Thorne, 2006), donde se entiende la cognición como una actividad social mediada por herramientas simbólicas construidas culturalmente, como el lenguaje o los conceptos. El uso del lenguaje, por tanto, no supone la representación de un pensamiento previamente elaborado, sino que su uso transforma y reorganiza los procesos mentales, tanto los propios como los de los individuos a quienes va dirigido. Así, la concienciación metapragmática en forma de habla metapragmática es entendida en esta investigación a partir del concepto de languaging, actividad en la que el lenguaje funciona como mediación en el proceso de resolución de tareas complejas. Además, en esta actividad se crea el significado, se moldea el conocimiento y la experiencia, se facilita la solución de problemas y se alcanza un mayor conocimiento del objeto estudiado (Swain, 2006; Swain y Watanabe, 2013). En palabras de Swain (2006, p. 98) la actividad de languaging es:

(...) the process of making meaning and shaping knowledge and experience through language. It is part of what constitutes learning. Languaging about language is one of the ways we learn language. This means that the languaging (the dialogue or private speech) about language that learners engage in takes on new significance. In it, we can observe learners operating on linguistic data and coming to an understanding of previously less well understood material. In languaging, we see learning taking place.

El uso del lenguaje en forma de languaging, además de permitir alcanzar una mayor comprensión de aspectos lingüísticos, ayuda a considerar aspectos 
relacionados con la identidad en el uso de una nueva lengua, al reconstruir y reinterpretar el significado de las experiencias interculturales y alcanzar así una mayor comprensión de la propia identidad cultural (Lankiewicz y WąsikiewiczFirlej, 2014).

Asimismo, esta perspectiva teórica considera el aprendizaje como una actividad dinámica y social, donde en colaboración con otros estudiantes o con el profesor, los aprendices encuentran oportunidades de alcanzar un mayor conocimiento del fenómeno estudiado de lo que hubieran podido alcanzar de forma individual. El concepto de zona de desarrollo próximo (ZDP), propuesto por Vygotsky (1978), destaca la importancia de observar la actividad interaccional como forma de detectar las capacidades que están en proceso de desarrollo. La ZDP representa el lugar en el que se produce el aprendizaje, y hace referencia a "la diferencia entre lo que un individuo puede hacer por sí solo (evidencia de que algo ya está adquirido) y lo que puede hacer con ayuda de otras personas, es decir, aquello que está en proceso de ser adquirido” (Antón, 2010, p. 11). Así, siguiendo a esta autora, la participación colaborativa con otros individuos es fundamental en la creación de oportunidades que hagan avanzar al sujeto en la ZDP y desarrollar habilidades que puedan llegar a ser interiorizadas con el tiempo. De esta forma, el aprendizaje surge de la participación en el diálogo colaborativo (Swain y Watanabe, 2013) donde el lenguaje es empleado como herramienta de mediación cognitiva en la interacción con los demás.

Además, los conceptos, considerados sistemas de significado simbólico construidos culturalmente, también median y estructuran la actividad cognitiva humana (Vygotsky, 1986). Como forma de promover el desarrollo conceptual, la enseñanza basada en conceptos en L2 ha sido considerada por diferentes autores, para facilitar tanto el aprendizaje de aspectos gramaticales (Negueruela, 2003) como pragmáticos (van Compernolle, 2014). La enseñanza de conceptos, en combinación con actividades de verbalización, permite a los estudiantes realizar reflexiones en las que pueden expresar y construir el significado a través de las características conceptuales, en lugar de establecer asociaciones basadas en la adecuación de las normas que rigen su uso.

\subsection{Pragmática sociocultural: herramientas para el desarrollo de la conciencia metapragmática en ELE}

Como forma de promover la conciencia metapragmática e intercultural en el aula de ELE y presentar a los estudiantes herramientas conceptuales que les permitan interpretar la interacción verbal intercultural, se propone en este 
trabajo partir del estudio de la cortesía como fenómeno social y comunicativo desde una aproximación sociocultural, pragmática sociocultural (Bravo, 2002, 2004; Bravo y Briz, 2004). Este acercamiento teórico se caracteriza por abordar los aspectos sociales y culturales que se manifiestan en el uso de la lengua desde una óptica relativista y destaca la importancia de "describir el uso situado de los recursos comunicativos provistos por una lengua determinada dentro de su propio sistema socio-cultural” (Bravo, 2009, p. 32). Se considera pertinente esta aproximación al estudio de la cortesía verbal al proporcionar una visión de los comportamientos y valores sociales consensuados que se manifiestan en los patrones comunicativos de un grupo social específico, considerando las características sociales, culturales, contextuales e individuales. Además, el empleo de este conocimiento teórico y conceptual permite establecer relaciones entre el significado que el uso de la lengua adquiere en diferentes contextos comunicativos, en la $\mathrm{C} 1$ y la $\mathrm{C} 2$. Así, aun reconociendo la existencia de comportamientos comunicativos, actitudes y valores que son conocidos, aceptados y practicados en las diferentes comunidades de habla (Hernández Flores, 2004), esta perspectiva teórica permitirá a los estudiantes no entender estos comportamientos como normas fijas de interacción, sino como tendencias orientativas sujetas a variación dependiendo del contexto, grupo social y características individuales de los participantes. Tal y como señala Bravo (2002), la importancia de esta aproximación está en "no tomar modelos a priori y regresar en cambio a las fuentes sociales del/la hablante, es decir a las convenciones compartidas dentro del grupo social de pertenencia, lo cual determina una particular concepción y configuración tanto de la imagen básica como de las imágenes de los roles que los hablantes desempeñan” (p. 144).

De forma específica, con el fin de fomentar la práctica reflexiva metapragmática y emplear los conceptos como herramienta de mediación en la creación de significado intercultural, se hace fundamental centrar la atención en algunos de los conceptos básicos empleados desde esta perspectiva teórica. Así, de forma específica, en este estudio se consideran los conceptos de imagen social básica e imagen del rol (Bravo, 2002, 2004) que, junto con el concepto de contexto, permiten delimitar las características socioculturales específicas y determinar los comportamientos corteses en una determinada comunidad de habla (Bravo, 2004; Bernal, 2007). Se consideran también los conceptos de autonomía y afiliación como forma de determinar los contenidos de imagen de cada sociedad particular (Bravo, 2005). Aunque estos conceptos son considerados universales, son entendidos como categorías vacías de contenido que deben rellenarse con los significados socioculturales específicos en cada contexto comunicativo específico (Bravo, 2008). Finalmente, ya que desde la pragmática sociocultural se indica la importancia 
de entender el efecto interpersonalmente positivo que el uso de la cortesía tiene en la interacción comunicativa (Hernández Flores, 2002; Bravo, 2005), se estima relevante el concepto de efecto social. Esta noción es clave en la interpretación de enunciados verbales y esencial para entender la cortesía valorizante, dirigida a confirmar la imagen social y estrechar las relaciones sociales entre los participantes (Albelda y Briz, 2010; Barros García, 2011; Hernández Flores, 2013).

El empleo de esta perspectiva teórica, ha sido utilizada en diferentes investigaciones para definir algunas de las premisas socioculturales más sobresalientes de la imagen social española (Hernández Flores, 2002; Bernal, 2007), donde se señala cómo la imagen de autonomía se caracteriza por la autoafirmación y la imagen de afiliación por el deseo de establecer una relación de confianza y familiaridad. Así, se hace relevante entender la cortesía no solamente como la realización de acciones mitigadoras de posibles actos amenazadores de la imagen sino también en cuanto a acciones valorizantes de la imagen (Albelda, 2005; Barros García, 2011). En cuanto a la imagen social escandinava, Henning (2015) indica que la autonomía se caracteriza por mostrarse como una persona independiente y no interferir en el espacio privado de los demás. Por otro lado, la afiliación se manifiesta mostrando consenso y compartiendo los intereses de los demás como parte de un colectivo.

\section{Marco metodológico}

\subsection{Contexto y participantes}

Este estudio es parte de una investigación más extensa (Morollón Martí, 2016) donde se muestra el diseño, la implementación y la evaluación de una intervención didáctica dirigida a promover la concienciación sociopragmática en L2 entre estudiantes universitarios daneses de español como lengua extranjera (ELE) en la Universidad de Aarhus ${ }^{2}$ (UAA), Dinamarca. En esta intervención, empleando una metodología de aprendizaje mixto (Helm, 2013), tres tipos de sesiones se suceden a lo largo de un semestre académico de doce semanas: (1) sesiones de telecolaboración sincrónica entre los

2 Los estudiantes daneses cursaban estudios universitarios de Lengua, Literatura y Cultura Española e Hispanoamericana. 
estudiantes daneses y estudiantes hablantes nativos de español de la Facultad de Filología de la Universidad de Alicante (UA); (2) sesiones de instrucción explícita centradas en la enseñanza de cortesía desde una perspectiva sociocultural; y (3) sesiones de reflexión en grupos entre los estudiantes daneses (ver Figura 1).

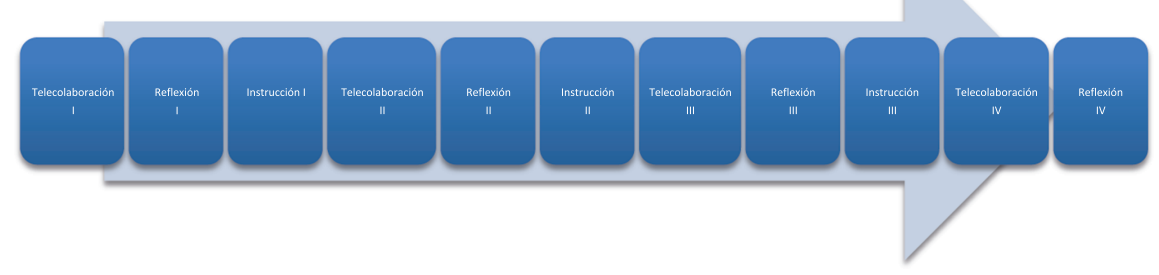

Figure 1: Implementación cronológica de los diferentes tipos de sesiones.

Para mostrar de qué forma los estudiantes emplean el conocimiento teórico en la interpretación de sus interacciones interculturales telecolaborativas, este trabajo se centra en el análisis de las sesiones intraculturales de reflexión en grupos ${ }^{3}$. Para ello, se empleó una metodología basada en los datos de los aprendices (Vyatkina, 2012), lo que permitió a los propios estudiantes delimitar las características contextuales y situacionales específicas de la situación comunicativa en la que ellos mismos habían participado. Como forma de estructurar estas reflexiones, la profesora del curso facilitó una lista con preguntas que podían emplear de forma orientativa. De forma específica, estas preguntas guiaban a los estudiantes a interpretar el significado social de los actos comunicativos de los saludos, despedidas, halagos, ofertas e invitaciones. Como muestra la Tabla 1, el contenido teórico se distribuyó en tres sesiones a lo largo del semestre. Puesto que la intervención se implementó en un contexto universitario, se consideró relevante introducir el estudio de la cortesía desde una visión histórica e incluir la teoría propuesta por Brown y Levinson (1987). La presentación de esta teoría sirvió como punto de partida para mostrar posteriormente la propuesta de relatividad cultural adoptada por la pragmática sociocultural.

Dos grupos de estudiantes participaron en este estudio: estudiantes de primer semestre de grado y segundo semestre de máster. Debido a la inestabilidad de participación, se seleccionaron los datos de los estudiantes que habían

3 A elección de los estudiantes, estas sesiones de reflexión en grupos se realizaron en danés con los estudiantes de grado y en español con los estudiantes de máster. 
Tabla 1: Contenido teórico presentado en las sesiones de instrucción explícita. (Adaptado de Morollón Martí y Fernández, 2016, p. 137).

\begin{tabular}{|c|c|}
\hline & Resumen de contenido \\
\hline Sesión I & $\begin{array}{l}\text { - ¿Qué es la pragmática? } \\
\text { - ¿Qué es un acto comunicativo? ¿Cómo podemos identificarlo? } \\
\text { - } \quad \text { Importancia de considerar los conceptos de: contexto, participantes, } \\
\text { relación entre los participantes y rol. }\end{array}$ \\
\hline Sesión II & $\begin{array}{l}\text { - } \quad \text { Introducción a la teoría de la cortesía desde una perspectiva histórica: la } \\
\text { - } \quad \text { Estudio de la cortesía desde una perspectiva sociocultural (definición de } \\
\text { Bravo, 2005). } \\
\text { - } \quad \text { Conceptos de autonomía y afiliación (Bravo, 2002, 2005). } \\
\text { - } \quad \text { Concepto de imagen social (Goffman, 1967). } \\
\text { - } \quad \text { Equilibrio en los deseos de imagen (Hernández Flores, 2002). } \\
\text { - } \quad \text { Actos corteses sin amenaza (Albeda y Briz, 2010). }\end{array}$ \\
\hline Sesión III & $\begin{array}{l}\text { - Cortesía atenuadora y cortesía valorizante. } \\
\text { - } \quad \text { Sociopragmática: consecuencia-efecto social. } \\
\text { - } \quad \text { Rol: definido culturalmente. } \\
\text { - Estudios sobre cortesía en español peninsular (Bravo, 2002, 2005; Albelda } \\
\text { y Briz, 2010; Hernández Flores, 2002). } \\
\text { - Relaciones sociales en español peninsular: solidaridad, familiaridad, } \\
\text { afiliación y cercanía. }\end{array}$ \\
\hline
\end{tabular}

participado en todas las sesiones a lo largo del semestre académico: 7 estudiantes de la primera intervención didáctica y 4 de la segunda. Estos 11 estudiantes eran hablantes nativos de danés, de edades comprendidas entre 20 y 25 años, y reconocieron, al comienzo del semestre, no tener ningún conocimiento teórico sobre pragmática.

\subsection{Aproximación metodológica}

Esta investigación adopta una metodología de estudio de caso cualitativo (Merriam y Tisdell, 2015), de naturaleza exploratoria e interpretativa que permite alcanzar una mayor comprensión sobre la naturaleza y el proceso de aprendizaje de lenguas y de la participación de los estudiantes en la actividad interpretativa. Más allá de buscar una generalización de los resultados, el estudio de caso permite realizar un análisis profundo y detallado de las interpretaciones realizadas durante el desarrollo de las reflexiones en grupo. Además, se adopta una perspectiva microgenética al análisis de los datos (Wersch, 1985), que permite 
detectar el origen y el proceso de desarrollo cognitivo en el contexto histórico y cultural en el que este tiene lugar. Esta perspectiva posibilita observar la formación de un proceso en desarrollo y no solamente el resultado del mismo (Lantolf y Thorne, 2006) e identificar los momentos considerados significativos en la actividad de concienciación metapragmática durante la interacción social. El empleo de esta perspectiva en el análisis de los datos no pretende mostrar el resultado del aprendizaje obtenido como un producto estático, sino analizar la actividad de reflexión in situ con el propósito de alcanzar una mayor comprensión de cómo los conceptos teóricos son empleados en la construcción del significado.

\subsection{Recogida y procedimiento de análisis de datos}

Las sesiones de reflexión en grupos fueron grabadas en audio y transcritas en su totalidad por la profesora del curso y autora de este trabajo, siguiendo el sistema de transcripción presentado en el anexo 1. El corpus de reflexiones con las que se trabajó forma un total de 9 horas y 1 minuto. Las grabaciones se realizaron con el permiso de los estudiantes y se sustituyeron sus nombres por pseudónimos en la presentación de los datos para mantener el anonimato. Este material fue transcrito y codificado en NVivo10 ${ }^{4}$ empleando la unidad de análisis denominada episodios de reflexión metapragmática (ERMP). Los ERMP han sido definidos como las partes de las reflexiones en grupos en las que los estudiantes, haciendo uso del contenido teórico y conceptual introducido en las sesiones de instrucción explícita, participan en una actividad de creación de significado donde "hacen referencia, cuestionan o reflexionan sobre aspectos sociopragmáticos y su aparición en las sesiones de telecolaboración u otras situaciones de comunicación intercultural” (Morollón Martí, 2016, p. 114). La codificación de los ERMP se realizó en base a dos categorías relacionadas entre sí: ERMP relativos a los actos comunicativos sobre los que reflexionan (esto es, saludos, despedidas, halagos, ofertas e invitaciones) y ERMP en los que los estudiantes emplean los conceptos teóricos presentados en las sesiones de instrucción explícita en la interpretación de tales actos comunicativos. De esta forma, en el análisis de los datos se puede interpretar en relación a qué acto comunicativo se está utilizando el significado conceptual. La cuantificación de

4 NVivo 10: software dirigido a la investigación con métodos cualitativos y mixtos que permite organizar, analizar y encontrar diferentes perspectivas en datos no estructurados o cualitativos. (http://www.qsinternational.com/Languagepages/Spanish). 
los ERMP encontrados según el acto comunicativo que interpretan y el concepto teórico empleado en su interpretación queda representado en la Tabla 2.

Es importante indicar que el empleo de los conceptos pragmáticos puede aparecer superpuesto a lo largo de las reflexiones, por lo que los ERMP también se superponen. Así, el concepto de rol aparece frecuentemente en relación con la delimitación de las características contextuales y su uso se da también en relación con la interpretación del efecto social que produce la realización del halago. Debe también considerarse la longitud variable y las características intrínsecas o cualidades de cada uno de los ERMP codificados. A este respecto, un ERMP puede estar formado por una única intervención o ser coconstruido en sucesivas intervenciones por varios de los participantes. Además, en cuanto a la cualidad de los episodios, estos pueden mostrar tanto un uso explícito del concepto pragmático en cuestión como una simple mención sin explicación o un uso implícito de los mismos. Así, el concepto de afiliación y su manifestación mediante la confianza y cercanía en la comunidad de habla española puede emplearse de forma explícita o puede ser discutido usando conceptos cotidianos como "los españoles son más abiertos" o "se le da muy bien eso de mantener la conversación en marcha". De esta forma, debe indicarse la naturaleza única de cada uno de los ERMP analizados, los cuales, además, deben entenderse en relación a las reflexiones previas y a las experiencias interculturales telecolaborativas de cada uno de los participantes.

Tabla 2: Categorización y cuantificación de los ERMP.

ERMP - Referencias a actos comunicativos

\begin{tabular}{llll}
\hline Saludo & Despedida & Halago & Oferta e invitación \\
18 & 21 & 28 & 30 \\
\hline
\end{tabular}

ERMP - Empleo de conceptos pragmáticos en la interpretación de los actos comunicativos

\begin{tabular}{lllll}
\hline Afiliación & Autonomía & Imagen & Amenaza & Atenuación \\
17 & 5 & 11 & 8 & 17 \\
Cortesía & Efecto social & Contexto & Rol & Valorización \\
29 & 23 & 51 & 43 & 23 \\
\hline
\end{tabular}

Para asegurar la fiabilidad en el proceso de codificación de los datos, se realizó un test de intercodificación donde, a partir de los ERMP mostrados en la tabla 2, un 
segundo investigador codificó un $20 \%$ del total del material analizado. A partir de la primera codificación, en la que se obtuvo un índice Kappa ${ }^{5}$ entre 0 y 1, se discutieron y redefinieron las categorías con un índice inferior a 0,4. Un segundo test de intercodificación mostró un índice Kappa entre 0,6 y 1. La revisión de las categorías con un nivel bajo de acuerdo reveló que las discordancias que se producían en la delimitación de los ERMP estaban relacionadas con el momento en que comenzaban o terminaban los episodios y se consideró que esta variación no afectaba al análisis de los datos.

Con el propósito de mostrar de qué forma se manifiesta la conciencia metapragmática durante el desarrollo de estas sesiones en grupos, se seleccionaron los ERMP en los que los estudiantes construyen el significado de los actos comunicativos del saludo, despedida, halago, oferta e invitación. Además, los ERMP mostrados en este estudio se han seleccionado por considerarse representativos del total de ERMP analizados y por evidenciarse en ellos de forma clara la interpretación realizada a través de los conceptos teóricos de cortesía, autonomía y afiliación y su relación con los conceptos de contexto, rol, efecto social y relación entre los participantes.

Ya que se considera en esta investigación el desarrollo de concienciación metapragmática como una actividad mediada por la participación en la intervención didáctica y los tres tipos de sesiones que la conforman, no deben entenderse los ERMP mostrados en este trabajo como el resultado de una relación causal entre la enseñanza y el aprendizaje. Así, estos deben comprenderse, más bien, como el resultado parte de una actividad mediada por el conocimiento teórico y conceptual, por la participación en las demás sesiones que forman la intervención didáctica y por las intervenciones de los demás participantes del grupo en el que se realiza la reflexión.

\section{Análisis de los datos y discusión}

El análisis de los ERMP muestra la importancia que para los estudiantes tiene la reconstrucción de las experiencias interculturales obtenidas en las sesiones de telecolaboración. Esto se evidencia en la descripción y contextualización que los estudiantes hacen de la situación comunicativa que es objeto de análisis, donde

5 El coeficiente Kappa de Cohen es una medida estadística empleada para calcular la fiabilidad en la intercodificación que ajusta el efecto del azar en los datos observados. El software de análisis NVivo10 empleado señala como acuerdos pobres los resultados por debajo de 0,40, acuerdos favorables los resultados entre 0,40 y 0,74 , y acuerdos excelentes los resultados por encima de 0,75 . 
se detallan las características situacionales y de los roles de los participantes en la interacción. Esta contextualización, esencial en cualquier análisis pragmático de la comunicación verbal, además de delimitar las características comunes a las conversaciones telecolaborativas y las especificidades de cada una de ellas, permite a los estudiantes participar en una actividad de reconstrucción de sus experiencias interculturales. Por otro lado, el empleo del lenguaje en interacción social permite crear oportunidades de coconstrucción del significado del uso de la lengua en tales intercambios desde una perspectiva pragmática. Además, el uso de conceptos relativos a la pragmática sociocultural proporciona oportunidades de emplear un lenguaje metapragmático a través del cual los estudiantes alcanzan una visión del lenguaje más allá del mero intercambio de información. Como muestran las siguientes secuencias, estos conceptos permiten a los estudiantes entender los intercambios comunicativos en cuanto al efecto social que su uso supone en la interacción y en cuanto a los valores sociales y culturales que se manifiestan en dichos intercambios.

La secuencia 1 muestra de qué forma los estudiantes interpretaron el acto comunicativo del halago. De forma específica, el halago más habitual encontrado en las conversaciones telecolaborativas es el realizado por los estudiantes de la UA en relación con la capacidad lingüística en español de los estudiantes de la UAA.

\section{Secuencia 1}

Interpretación del acto comunicativo del halago a través de los conceptos de rol, efecto social y cortesía valorizante. Intervención didáctica $1^{6}$.

1 Gitte: $\quad$ sí, es justo por eso, pienso, para hacer al no hablante de español más seguro ehhh, y así para hacerle saber, ok, puedo hacer algún fallo, que no pasa nada, y ehhh el hablante de español dice, sí, sí, está bien, te entiendo muy bien y cosas de ese estilo, y se queda uno así, ehhh, que te confirman, bueno está bien

2 Astrid: sí, bueno tiene algunas consecuencias buenas

(...)

3 Astrid: pero ese ejemplo es así, bueno, hacen que la conversación sea más tranquila porque se confirma en, en eso de lo que acabamos de hablar

4 Gitte: $\quad$ sí

5 Ida: $\quad$ también es porque el danés ha estado un poco nervioso

6 Esta reflexión en grupo se realizó en danés. Con el objetivo de ganar claridad en la presentación de los datos, se ha optado por mostrar únicamente la traducción al español, realizada por la autora de este estudio. 
$\begin{array}{ll}6 \text { Astrid: } & \text { sí } \\ 7 \text { Ida: } & \text { donde ella o él ha } \\ 8 \text { Gitte: } & \text { sí } \\ 9 \text { Ida: } & \text { y un poco inseguro }\end{array}$

Como muestra la secuencia $1^{7}$, durante la interpretación del acto comunicativo del halago, las estudiantes hacen referencia al rol de los participantes en la interacción en relación con la habilidad lingüística de la lengua empleada (el español) como forma de diferenciar entre el rol de hablante nativo y hablante no nativo (turno 1). Además, resaltan la desigualdad que existe entre los participantes e indican que, por esta razón, el estudiante danés estaba nervioso y se sentía inseguro (turnos 5 y 9). De esta forma, la delimitación de las características relativas al rol de los participantes son empleadas para demarcar las razones por las que consideran que se realizó el halago. Este aspecto es fundamental para evaluar el efecto interpersonal que la realización del acto tiene en la interacción (Bernal, 2007). Así, indican que, al efectuar el halago, el estudiante de la UA confirma que entiende lo que el estudiante de la UAA expresa, sintiéndose este último más seguro (turnos 1 y 3 ). Asimismo, añaden que su realización tiene consecuencias positivas en la interacción en la medida en que ayuda a que esta fluya más tranquila (turnos 2 y 3). De esta manera, las estudiantes muestran conciencia de cómo el empleo de cortesía valorizante confirma la imagen social de los participantes y estrecha las relaciones sociales (Albelda y Briz, 2010).

De esta forma, las estudiantes revelan su capacidad para realizar interpretaciones pragmáticas sobre el uso de la lengua al establecer relaciones entre las características contextuales, el rol de los participantes, la realización del halago y el efecto social que este tiene en la interacción. Aunque los conceptos de contexto, rol y efecto social no son empleados de forma teórica, ni son verbalizados explícitamente, el significado de los mismos es integrado en la reflexión, mediando la actividad de concienciación metapragmática.

Al acudir al significado conceptual presentado en las sesiones de instrucción explícita, el análisis de los ERMP muestra la capacidad de los

7 Las preguntas a partir de las que surge este ERMP son: En la mayoría de las conversaciones, los estudiantes de la UA han realizado halagos a los estudiantes de la UAA. ¿Por qué crees que se realizaron? ¿Qué consecuencia/efecto social crees que tuvo el halago en la conversación? ¿Qué factores contextuales son importantes de tener en cuenta para entender los halagos en estas conversaciones? 
estudiantes para interpretar el significado de determinadas acciones realizadas en sus conversaciones y relacionar este significado con los actos comunicativos trabajados durante la intervención didáctica. Los estudiantes muestran así su capacidad para transferir el significado conceptual a otras situaciones comunicativas. Este tipo de reflexiones se evidencia en la secuencia 2, donde a partir de una interpretación de las invitaciones y las ofertas ${ }^{8}$ realizadas durante las sesiones de telecolaboración, se establece una relación con otro tipo de acción realizada durante la despedida.

\section{Secuencia 2}

Interpretación del acto comunicativo de la despedida a través de los conceptos de efecto social y cortesía valorizante. Intervención didáctica 2.

1 Katrine: $\quad$ yo creo que una cosa que es bastante interesante en mi conversación y no sé exactamente dónde ponerlo en, en, en estas estrategias y actos de habla y todo eso, pero cada vez que nos despedimos es como, que nos invitamos por ejemplo a leer algún artículo, o nos mandamos un link de un video y algo y, siempre para la próxima vez, hemos leído o hemos visto el video y reflejado un poquito sobre eso y podemos hablar otra vez del tema, ¿no? que por ejemplo nos habíamos mandado vídeos de poetas y, y, hablábamos bastante de eso, ¿no? y, y, es para mí un

2 Cecilie: sí, pero ustedes se salen bastante del contexto de

3 Katrine: sí

4 Cecilie: de investigación de estudiante, del contexto profesional

5 Katrine: pero también siempre estamos en el contexto de lengua

6 Cecilie: sí, sí

7 Katrine: $\quad$ y es como, bueno, se puede salir mucho, siempre nos quedamos ahí ¿no? ella

8 Cecilie: $\quad$ porque son sus intereses, ¿no?

9 Katrine: $\quad$ exacto, sí

10 Cecilie: a lo mejor si tuvieran una conversación fuera de este contexto, estuvieran hablando de lo mismo

(risas)

\footnotetext{
8 Las invitaciones y ofertas en las que se centran las reflexiones son las realizadas por los estudiantes de la UA, en en las que invitan u ofrecen a los estudiantes de la UAA ir a visitarlos a Alicante, poder quedarse a dormir en sus casas si algún día van a Alicante o poder ponerse en contacto con ellos si visitan su ciudad.
} 
11 Katrine: sí (risas) yo creo que sí, pero no sé si, si, yo creo que, hasta cierto punto se puede hablar de un tipo de cortesía valorizante ¿no? que, que nos invitamos y, y mostramos a la vez que, que nos interesamos por lo que, que la otra persona ha, no sé cómo hablar

12 Cecilie: ha dicho

13 Katrine: sí, ella me manda algo y yo me intereso por lo que me ha mandado ¿no?

14 Hanne: sí, como afirmativo

15 Katrine: sí, siempre, exacto

16 Signe: pues no me da igual lo que me has entregado ¿no? me importa lo que me has dado entonces que yo voy a verlo, que yo voy a pensarlo

17 Hanne: $\quad$ y por eso em, tú me importas

18 Signe: $\quad$ sí, eso es lo mismo ¿no?

19 Hanne: sî

En la secuencia 1, Katrine (turno 1) relata que, durante la despedida, su compañera de telecolaboración de la UA y ella suelen enviarse algún vídeo o enlace que les gusta. A este respecto, Katrine establece una relación entre esta acción y las invitaciones (turno 11), al considerar que ambas son una forma de expresar cortesía valorizante, ya que con su realización se muestra interés hacia la otra persona (turnos 11 y 13). Esta reflexión es compartida por las demás participantes del grupo (turnos 14 al 19). Este ERMP muestra cómo estas estudiantes, en el desarrollo del diálogo colaborativo, participan en una actividad de construcción del significado mediada por el uso del habla metapragmática y el concepto teórico de cortesía valorizante. El empleo del significado conceptual les permite, más allá de usar normas pragmáticas preestablecidas, interpretar diferentes tipos de acciones comunicativas que puedan aparecer en sus conversaciones interculturales. De esta forma, se muestra cómo el concepto adquiere funcionalidad teórica y práctica (Negueruela, 2003). En alusión a ello, debe subrayarse la importancia de no entender la funcionalidad práctica en este estudio únicamente como la realización de acciones por parte de los estudiantes que impliquen cortesía valorizante, sino como la participación en una actividad de reflexión significativa en la que el concepto es integrado en la creación de significado en situaciones comunicativas interculturales.

También los conceptos de cortesía, autonomía y afiliación son empleados en diferentes ERMP como forma de interpretar las prácticas comunicativas observadas en las dos comunidades de habla, la danesa y la española, sobre las que reflexionan las estudiantes. 


\section{Secuencia 3}

Interpretación del concepto de cortesía y su relación con las características del contexto sociocultural a través de las categorías de autonomía y afiliación. Intervención didáctica 2.

1 Hanne: porque la, así como hemos hablado que, que, la cortesía también tiene, depende mucho de los hablantes del contexto eh, sociocultural y todo eso que se gira sobre el contexto

2 Cecilie: la respuesta está prácticamente en la introducción ¿no?

3 Hanne: sí (risas)

4 Cecilie: desempeñar el rol específico según lo esperado en un contexto determinado ¿no? entonces que, si no sabemos cómo es el contexto, no sabemos cómo comportarnos

5 Hanne: claro

6 Signe: yo creo trata de lo que hemos nombrado antes, eso de afiliación y valori-, no, ¿cómo se dice?

7 Cecilie: afiliación y autonomía

8 Kirstine: la autonomía, sí

9 Signe: Sí, eso, entonces el contexto es importante si estamos en, pues en un contexto de autonomía como en Dinamarca o, dominante la autonomía, entonces se tiene que comunicarse de una manera distinta, por supuesto, entonces si vas a comunicar en un contexto de más afiliación, entonces

10 Cecilie: sí, o sea, concebimos el, la cortesía de forma diferente ¿no?, como hablamos la, la vez pasada, que para nosotros ${ }^{9}$ de, de repente, se es cortés ofrecer que tú te puedes quedar en la casa de no sé quien

11 Katrine: sí

12 Cecilie: si al final no es así

13 Katrine: sí, (risas) exacto

14 Cecilie: mientras, desde el punto de vista de la otra persona es al revés ¿no?

15 Katrine: sí, porque

16 Cecilie: entonces depende de

17 Katrine: si hablamos de, de la cortesía atenuadora y la cortesía valorizante, parece que, parece que los hispanohablantes utilizan mucho más la valorizante

18 Cecilie: $\mathrm{mhm}$

9 Se entiende el uso de nosotros en esta reflexión en sentido figurado, ya que se interpreta que Cecilie en esta intervención está haciendo referencia a las invitaciones realizadas por los estudiantes de la UA y que surgieron en la mayoría de las conversaciones telecolaborativas. 
19 Katrine: mientras nosotros estamos atenuando todo el tiempo para no 20 Signe: cuídate, tu imagen (risas)

(...)

21 Katrine: pero eso es una manera de, pues, que yo he visto un montón de veces con hispanohablantes que es, la voluntad sí está ¿no?, que realmente quieren, ay sí, quieren como, sí, dar una buena impresión y hacer una, una

22 Cecilie: dar una buena impresión es fácil (risas)

23 Katrine: crear un buen ambiente y luego no pueden cumplirlo porque le ha pasado algo o lo había olvidado y, y no sé si, porque para nosotros, es como, que nos ofende, ¿no?

24 Signe: es un choque ¿no? un choque cultural

Como muestra la secuencia $3^{10}$, los conceptos de cortesía, autonomía y afiliación se relacionan con las características contextuales, los valores socioculturales y el rol de los participantes (turnos 1, 4, 6 y 9). Las estudiantes muestran conciencia de la relatividad que supone el empleo de estos conceptos en diferentes comunidades de habla. De forma específica, indican (turnos 10 al 16), con respecto a la realización de las invitaciones en sus conversaciones telecolaborativas, que en diferentes contextos culturales debe entenderse la cortesía de diferente forma. Del mismo modo, Katrine señala (turno 17), haciendo referencia a los conceptos de cortesía atenuadora y cortesía valorizante, el tipo de cortesía que ella considera más empleada en la cultura hispanohablante y en la danesa (turno 19). Además, el valor de esta reflexión reside en la capacidad que en ella se muestra de asociar la situación comunicativa analizada a experiencias interculturales previas. Así, esta estudiante indica haber observado una tendencia a realizar diferentes tipos de ofertas que, por diferentes razones no llegan nunca a cumplirse (turno 21 y 23), un comportamiento social que ella considera ofensivo desde su C1. Finalmente, y a partir de estas reflexiones, se indica (turno 24) que es a causa de estas diferencias por lo que tiene lugar el choque cultural, es decir, se producen malinterpretaciones con respecto al significado que estos comportamientos tienen en diferentes comunidades de habla. De esta forma, tal y como apunta Briz (2004), la familiaridad que se muestra en las interacciones comunicativas en España

10 Las preguntas presentadas por la profesora a partir de las que surge este ERMP fueron formuladas así: Ya has hablado con el estudiante de la UA cuatro veces. ¿De qué forma crees que esto se refleja en la conversación? ¿Cómo consideras que ha sido la participación en estas conversaciones de telecolaboración? ¿Crees que eres más consciente del uso que se hace de la cortesía en diferentes situaciones comunicativas? ¿Crees que hay factores culturales que pueden influir en la conversación? ¿Crees que puedes usar este conocimiento de ahora en adelante? 
puede ser interpretada por personas de otras culturas como un comportamiento descortés.

En este ERMP se evidencia la conciencia sociopragmática que estas estudiantes han alcanzado del concepto de cortesía y su uso por parte de hablantes nativos de español y hablantes daneses. Esto coincide con lo señalado en investigaciones empíricas, donde a partir de una comparación en el uso de cortesía en interacciones entre hablantes españoles y escandinavos, se aprecia una mayor frecuencia de uso de estrategias de cortesía valorizante entre los hablantes de español (Bernal, 2012). Esto se manifiesta mostrando confianza y familiaridad (Bernal, 2007) no solo entre personas cercanas, sino también entre personas desconocidas (Hernández Flores, 2002; Briz, 2004). Esta confianza interpersonal, considerada una de las premisas culturales más destacadas en la comunidad de habla española, se relaciona con la familiaridad e intimidad que se muestra en la interacción verbal. Está permitido hablar sin temor de ofender al interlocutor y se realizan actos valorizadores de la imagen como halagos o invitaciones con los que se muestra aprecio, se es solidario y se establece un clima de cercanía social (Bernal, 2007; Barros García, 2011). Además, en esta actividad de creación de significado se establecen relaciones entre los comportamientos sociales observados en ambas comunidades de habla. De esta forma, se establece una comparación interpretativa entre la C1 y la C2, aspecto que ha sido considerado esencial en el desarrollo de la competencia intercultural (Dervin y Liddicoat, 2013) y en el desarrollo de la conciencia metapragmática desde una perspectiva intercultural (McConachy, 2013).

Finalmente, es relevante señalar cómo, durante el desarrollo de las reflexiones, los estudiantes consideran la utilidad del conocimiento teórico presentado en las sesiones de instrucción explícita como forma de interpretar las prácticas verbales que surgen en la interacción intercultural. El análisis de este tipo de ERMP indica una mayor conciencia de la aplicabilidad de este conocimiento teórico entre las estudiantes de la segunda intervención didáctica que entre los estudiantes de la primera. Este hecho se atribuye a una mayor madurez académica y capacidad para valorar la aplicabilidad de dicho conocimiento. Además, este tipo de episodios muestra el reconocimiento por parte de los estudiantes del potencial de la pragmática sociocultural en la mediación de interpretaciones interculturales en diferentes contextos interaccionales.

Secuencia 4

Interpretación de la aplicabilidad del conocimiento teórico a situaciones comunicativas interculturales. Intervención didáctica 2.

1 Katrine: em, y de ahí creo que entiendo mejor la manera de pensar de la otra persona porque, porque tengo todas estas cosas en la cabeza 
2 Cecilie: para mí, el gran cambio no tanto es el, el ser consciente de, de, o sea de cómo es la otra persona y que hay diferencias culturales y todo eso, sino que ahora hemos caracterizado esas diferencias, es más el hecho de haberlo nombrado, porque aunque, yo creo que automáticamente somos conscientes de que ah, eso fue diferente, ¿no?

3 Katrine: claro

4 Cecilie: yo no lo haría así, pero ahora lo hemos caracterizado

5 Katrine: sí

6 Hanne: ya sabemos qué es

7 Cecilie: los términos

(....)

8 Cecilie: $\quad$ sí, para mí muchas cosas han sido la primera ¿no? lo que pasamos la vez pasada de, de, cortesía valorante* $\mathrm{y}$, atenuadora

9 Hanne: ya

10 Cecilie: para mí fue la primera ¿no? y fue buenísimo

(....)

11 Signe: pero en realidad la cuestión es entenderlo como un

12 Cecilie: como un concepto

13 Signe: sí

14 Cecilie: como se ha nombrado teóricamente todo es más fácil referirse a ello y

(...)

15 Signe: eso, pues para mí, eso de afiliación y valo-, no ¿cómo es? Otra vez me lo he

16 Cecilie: ¿cortesía valorizante?

17 Signe: autonomía y afiliación

18 Hanne: ah

19 Signe: $\quad$ eso para mí es lo más importante en realidad, eso de verse como dentro de un grupo y, y más de los lazos entre personas que, más como la persona en sí, eso, es un término es para pensarlo, creo, porque entonces puedes tener otras culturas y puedes tener esos términos y entonces, eso es más importante en esa cultura y eso, y tienes como, cosas para colocar, no sé

La secuencia 4 muestra con claridad la interpretación de los estudiantes de este estudio con respecto al conocimiento teórico relativo a la pragmática sociocultural. Son de especial relevancia las intervenciones de Cecilie y Signe en esta secuencia al asegurar que lo más importante no ha sido aprender un conocimiento nuevo, sino caracterizar y sistematizar ese conocimiento que de alguna forma ya intuían (turno 2). Por otro lado, reconocen que, aunque ellas no 
actuarían así en la interacción (turno 4), les ha sido útil establecer una relación entre los aspectos interculturales observados y los conceptos teóricos (turno 7), lo que les permite referirse más fácilmente a estos (turnos 11 al 14). También, más adelante (turnos 15 al 19), Signe señala la importancia que para ella tienen los conceptos de autonomía y afiliación e indica su utilidad en situaciones de comunicación intercultural en las que estos conceptos pueden ser relacionados con diferentes comportamientos comunicativos, e indica también que es una pena no haberlos trabajado en cursos anteriores durante sus estudios.

De esta manera se muestra en este ERMP cómo introducir a los estudiantes de ELE a los conceptos más relevantes empleados en estudios desarrollados en el marco teórico de la pragmática sociocultural les permite interpretar los comportamientos comunicativos interculturales desde una perspectiva de relatividad cultural. Además, ya que esta perspectiva teórica considera los factores sociales y culturales específicos de cada comunidad de habla, los estudiantes podrán emplear este conocimiento en otras interpretaciones interculturales, más allá de las realizadas en el contexto del aula.

\section{Conclusión}

En este trabajo se ha investigado el potencial de la enseñanza de (des)cortesía desde una perspectiva sociocultural en el aula de L2 como forma de promover la conciencia metapragmática a través de la práctica reflexiva y de integrar una dimensión intercultural en el aula de ELE. Partiendo del análisis de reflexiones intraculturales en grupo en un contexto de educación formal universitaria, se ha mostrado de qué forma los estudiantes daneses, participando en una actividad de languaging en forma de diálogo colaborativo, establecen relaciones entre su experiencia intercultural y los conceptos teóricos presentados en el aula, en forma de enseñanza explícita. El análisis de los datos revela cómo los conceptos más relevantes empleados en el área de estudio de la pragmática sociocultural permiten a los estudiantes interpretar las acciones que se manifiestan en el intercambio comunicativo intercultural, entre hablantes nativos de danés y español. Así, estos conceptos no son empleados en forma de conocimiento estático, sino que su significado es utilizado de forma creativa en la creación de significado de las prácticas discursivas en las que estos mismos estudiantes han participado durante las sesiones de telecolaboración. Es importante señalar que, aunque no todos los participantes muestran un dominio teórico de los conceptos trabajados, la relevancia de participar en esta actividad reflexiva se estima que consiste en las oportunidades interpretativas que los estudiantes 
encuentran durante sus reflexiones. Se considera que el valor de este análisis consiste en la posibilidad de observar la actividad cognitiva de los estudiantes trabajando en la ZDP durante la reconstrucción y coconstrucción del significado de los comportamientos sociales que se manifiestan en los actos comunicativos estudiados. Así, desde la perspectiva de la TSC, la importancia de promover actividades de diálogo colaborativo, donde los estudiantes participan en una actividad social de reconstrucción del significado interaccional, está en suscitar una práctica reflexiva verbalizada donde el uso de los conceptos y su significado teórico sean empleados como herramientas de mediación en la interpretación de situaciones comunicativas reales.

Además, se considera que introducir a los estudiantes a la perspectiva teórica de la pragmática sociocultural contribuye a promover la conciencia metapragmática al facilitar la realización de interpretaciones contextualizadas de los recursos comunicativos utilizados en la interacción verbal dentro de su propio sistema sociocultural (Bravo, 2009, p. 32) y en relación con las comunidades de habla estudiadas. Al mismo tiempo, el empleo de esta perspectiva teórica promueve entre los estudiantes de ELE una visión de la interacción verbal como una actividad situada que debe interpretarse y reinterpretarse desde un relativismo social, cultural y situacional, en cada situación comunicativa específica.

Finalmente, deben reconocerse las limitaciones de este estudio y avanzar posibles futuras líneas de investigación. Una de las limitaciones está relacionada con las escasas posibilidades que este estudio presenta para determinar el grado de conciencia metapragmática alcanzado por cada uno de los estudiantes a partir de la implementación didáctica. Puesto que esta se muestra durante las reflexiones en grupo como una actividad coconstruida, carecería de significado analizar las intervenciones de cada uno de los participantes por separado. Futuras investigaciones deberían considerar la posibilidad de introducir actividades de reflexión verbalizadas e individuales como complemento a las reflexiones en grupo. Además, la realización de un cuestionario al comienzo y al final del semestre académico sería beneficioso para evaluar posibles cambios en la conciencia metapragmática de cada uno de los estudiantes. A este respecto, una opción sería incluir una adaptación del cuestionario de hábitos sociales propuesto en el proyecto de investigación COSOPRAG ${ }^{11}$. Tomando como inspiración la propuesta presentada por Bernal

11 El proyecto de investigación COSOPRAG desarrolla "un modelo de registro de comportamientos socio-pragmáticos que permita desarrollar categorías aptas para estudiar la producción lingüística proveniente de distintas variantes del español” http://edice.org/cosoprag/ 
y Hernández Flores (2016), la aplicación de este cuestionario podría servir de punto de partida en el desarrollo de reflexiones metapragmáticas dentro del aula. Finalmente, futuras investigaciones deberían considerar en la enseñanza de la pragmática sociocultural los principios metodológicos de la enseñanza basada en conceptos como forma de promover el desarrollo conceptual (Negueruela, 2003). Así, tomando como punto de partida las reflexiones realizadas por los estudiantes, sería conveniente investigar de qué forma los conceptos pragmáticos trabajados adquieren funcionalidad práctica en situaciones comunicativas interculturales con hablantes nativos de español.

\section{Referencias}

Albelda, M. M. (2005). El refuerzo de la imagen social en la conversación coloquial del español. En D. Bravo (Ed.), Cortesîa lingüîstica y comunicativa en español. Categorîas conceptuales y aplicaciones a corpus orales y escritos, Buenos Aires, Dunken, 93-118.

Albelda, M. y Briz, A. (2010). Aspectos pragmáticos. Cortesía y atenuantes verbales en las dos orillas a través de muestras orales. En M. J. Aleza y M. Enguita (Eds.), La lengua española en América: normas y usos actuales (pp. 237-260). Valencia: Universidad de Valencia.

Alcón Soler, E. y Martínez-Flor, A. (2008). Pragmatics in Foreign Language Contexts. En E. Alcón Soler y A. Martínez-Flor (Eds.), Investigating Pragmatics in Foreign Language Learning, Teaching and Testing (pp. 3-21). Bristol: Multilingual Matters.

Antón, M. (2010). Aportaciones de la teoría sociocultural al estudio de la adquisición del español como segunda lengua. RESLA, 23, 9-30.

Barros García, M. J. (2011). La cortesía valorizadora en la conversación coloquial española: estudio pragmalingüístico. Tesis doctoral. Facultad de Filosofía y Letras. Departamento de Lengua Española: Universidad de Granada.

Bernal, M. (2007). Categorización sociopragmática de la cortesía y de la descortesía. Un estudio de la conversación coloquial española. Tesis doctoral. Estocolmo: Universidad de Estocolmo. Recuperado de: http://su.divaportal.org/smash/record.jsf?pid=diva2\% 3A196989\&dswid $=-4254$

Bernal, M. (2012). Ciberentrevistas y actividades de (des)cortesía. Un estudio comparativo español-sueco. Foro hispánico: revista hispánica de Flandes y Holanda (ejemplar dedicado a: Pragmática y comunicación intercultural en el mundo hispanohablante), 44, 159-186.

Bernal, M. y Hernández Flores, N. (2016). Variación sociopragmática en la enseñanza del español: aplicación didáctica de un cuestionario de hábitos sociales. Journal of Spanish Language Teaching, 3(2), 114-126.

Bravo, D. (2002). Actos asertivos y cortesía: imagen del rol en el discurso académico argentino. En M. E. Placencia y D. Bravo (Eds.), Actos de habla y cortesía en español. Londres: Lincom Studies in Pragmatics, 5, 141-174.

Bravo, D. (2004). Tensión entre universalidad y relatividad en las teorías de la cortesía. En D. Bravo y A. Briz (Eds.), Pragmática sociocultural: estudios sobre el discurso de cortesía en español (pp. 15-37). Barcelona: Ariel Lingüística. 
Bravo, D. (2005). Categorías, tipologías y aplicaciones. Hacia una redefinición de la cortesía comunicativa. (Ed.), En D. Bravo Estudios de la (des)cortesía en español. Categorías conceptuales y aplicaciones a corpora orales y escritos (pp. 21-52). Buenos Aires: Dunken.

Bravo, D. (2008). The implications of studying politeness in Spanish contexts: a discussion. Pragmatics, 18(4), 577-603.

Bravo, D. (2009). Pragmática, sociopragmática y pragmática sociocultural del discurso de la cortesía. Una introducción. En D. Bravo, N. Hernández Flores y A. Cordisco (Eds.), Aportes pragmáticos, sociopragmáticos y socioculturales a los estudios de cortesía en español (pp. 31-68). Buenos Aires: Dunken.

Bravo, D. y Briz, A. (Eds.). (2004). Pragmática sociocultural: estudios sobre el discurso de cortesía en español. Barcelona: Ariel Lingüística.

Briz, A. (2004). Cortesía verbal codificada y cortesía verbal interpretada en la conversación. En

D. Bravo y A. Briz (Eds.), Pragmática sociocultural: estudios sobre el discurso de cortesía en español (pp. 67-93). Barcelona: Ariel Lingüística.

Brown, P. y Levinson, S C. (1987). Politeness: Some universals in language usage. Cambridge: Cambridge University Press.

Byram, M. (2008). From foreign language education to education for intercultural citizenship: Essays and reflections. Clevedon: Multilingual Matters.

Cohen, D. A. y Sykes, J. M. (2013). Strategy based learning of pragmatics for intercultural education. En F. Dervin y A. J. Liddicoat (Eds.), Linguistics for Intercultural Education. (pp. 87-111). Amsterdam: John Benjamins Publishing Co.

Dervin, F. y Liddicoat, A. J. (Eds.). (2013). Linguistics for Intercultural Education. Amsterdam: John Benjamins Publishing Co.

Gironzetti, E. y Koike, D. (2016). Bridging the gap in Spanish instructional pragmatics: from theory to practice/Acortando distancias en la enseñanza de la pragmática del español: de la teoría a la práctica. Journal of Spanish Language Teaching, 3(2), 89-98.

Goffmann, E. (1967). Interactional Ritual: Essays on Face-to-face Behavior. Garden City, NY: Anchor Books.

Helm, F. (2013). A Dialogical Model for Telecollaboration. Bellaterra Journal of Teaching \& Learning Language \& Literature, 6(2), 28-48.

Henning, S. (2015). La construcción de la imagen social en dos pares adyacentes: Opiniónacuerdo/desacuerdo y ofrecimietno-aceptación/rechazo. Un estudio de la conversación familiar sueca y española. Tesis doctoral. Estocolmo: Universidad de Estocolmo.

Hernández Flores, N. (2002). La cortesía en la conversación española de familiares y amigos. La búsqueda del equilibrio entre la imagen del hablante y la imagen del destinatario. Tesis doctoral. Aalborg: Universidad de Aalborg.

Hernández Flores, N. (2004). La cortesía como la búsqueda del equilibrio de la imagen social. En D. Bravo y A. Briz (Eds.), Pragmática sociocultural: estudios sobre el discurso de cortesía en español (pp. 95-108). Barcelona: Ariel Lingüística.

Hernández Flores, N. (2013). Actividad de imagen, caracterización y tipología en la interacción comunicativa/Facework: characteristics and typology in communicative interaction. Pragmática Sociocultural, 2(1), 175-198.

Hymes, D. H. (1972). On communicative competence. En J. B. Pride y J. Holmes (Eds.), Sociolinguistics (pp. 269-293). Harmondsworth: Penguin.

Ishihara, N. y Cohen, A. (Eds.). (2010). Teaching and Learning Pragmatics. Where Language and Culture Meet (pp. 75-96). Edinburgh, United Kingdom: Pearson Education Limited. 
Kasper, G. y Rose, K.R. (2002). Pragmatic development in a second language. Oxford: Blackwell.

Kinginger, C. (2008). Language Learning in Study Abroad: Case Study of Americans in France. The Modern Language Journal, 92, Issue Supplement, 1-124.

Kinginger, C. y Farrell, K. (2004). Assessing development of metapragmatic awareness in study abroad. Frontiers. The Interdisciplinary Journal of Study Abroad, 10, 19-42.

Kramsch, C. (1993). Context and Culture in Language Education. Oxford: Oxford University Press.

Kramsch, C. (1993). Context and Culture in Language Education. Oxford: Oxford University Press.

Lankiewicz, H. y Wąsikiewicz-Firlej, E. (2014). Languaging Experiences: Learning and Teaching Revisted (1-32). New Castle: Cambridge Scholars Publishing.

Lantolf, J. y Thorne, S.L . (2006). Sociocultural Theory and the Genesis of Second Language Development. Oxford: Oxford University Press.

Liddicoat, A. J. (2008). Pedagogical Practice for Integrating the Intercultural in Language Teaching and Learning. Japanese Studies, 28(3), 277-290.

Liddicoat, A. J. y Scarino, A. (2013). Intercultural Language Teaching and Learning. New YorkOxford: Wiley-Blackwell.

McConachy, T. (2013). Exploring the meta-pragmatic realm in English language teaching. Language Awareness, 22(2), 100-110.

Merriam, S. B. y Tisdell, E. J. (2015). Qualitative Research. A Guide to Design and Implementation (4. Ed.). San Francisco: Wiley Brand.

Morollón Martí, N.S. y Fernández, (2016). Telecollaboration and Sociopragmatic Awareness in the Foreign Language Classroom. Innovation in Language Learning and Teaching - Online Intercultural Exchange, 10(1), 34-48.

Morollón Martí, N. (2016). Concienciación sociopragmática en el aula de español como lengua extranjera. Una aproximación sociocultural. Tesis doctoral. Aarhus: Universidad de Aarhus.

Negueruela, E. (2003). Systemic-Theoretical Instruction and L2 Development: A Sociocultural Approach to Teaching-Learning and Researching L2 Learning. Tesis doctoral. Pennsylvania State University.

Swain, M. (2006). Languaging, agency and collaboration in advanced second language profiency. En H. Byrnes (Ed.), Advanced Language Learning: The Contribution of Halliday and Vygotsky (pp. 95-108). London: Continuum.

Swain, M. y Watanabe, Y. (2013). Languaging: Collaborative Dialogue as a Source of Second Language Learning. En C. A. Chapelle (Ed.), The Encyclopedia of Applied Linguistics. Blackwell Publishing Ltd. doi: 10.1002/9781405198431.wbeal0664

Taguchi, N. (2015). Instructed pragmatics at a glance: Where instructional studies were, are, and should be going. Language Teaching, 48(1),1-50.

Thorne, S. L. (2003). Artifacts and cultures-of-use in intercultural communication. Language Learning and Technology, 7(2), 38-67.

Thorne, S. L. (2010). The "Intercultural Turn" and Language Learning in the Crucible of New Media. En F. Helm y S. Guth (Eds.), Telecollaboration 2.0 for language and intercultural learning (pp. 139-164). Bern, Switzerland: Peter Lang.

van Compernolle, R. A. (2014). Sociocultural Theory and L2 Instructional Pragmatics. Bristol, Buffalo y Toronto: Multilingual Matters. 
Vyatkina, N. A. (2012). Applying the methodology of learner corpus analysis to telecollaborative discourse. En M. Dooly y R. O’Dowd (Eds.), Researching Online Foreign Language

Interaction and Exchange: Theories, Methods and Challenges (pp. 267-303). Bern: Peter Lang.

Vygotsky, L. S. (1978). Mind in Society: The Development of Higher Psychological Processes. Cambridge, MA: Harvard University Press.

Vygotsky, L. S. (1986). Thought and Language. Cambridge, MA: MIT Press.

Wersch, J. V. (1985). Vygotsky and the Social Formation of Mind. Cambridge: Harald University Press.

\section{Anexo}

Sistema de transcripción

(( )) Fragmento indescifrable.

? Interrogaciones. También se utilizó para los apéndices del tipo ¿no?, ¿eh?, ¿sabes?

pala- Palabra inacabada.

(risas): Aparecen al final de cada intervención si solamente ríe el interlocutor que está hablando. En cambio, si ríen todos o varios participantes del grupo, figuran entre dos intervenciones.

Palabra* Incorrecciones gramaticales o semánticas.

Lista de abreviaturas

ELE Español como lengua extranjera

L2 Segunda lengua

C1 Propia cultura

C2 Cultura meta

TSC Teoría sociocultural

ZDP Zona de desarrollo próximo

ERMP Episodios de reflexión metapragmática

UA Universidad de Alicante

UAA Universidad de Aarhus 